\title{
Commentaire
}

\section{Metformin's contraindications should be contraindicated}

\author{
James McCormack, Kevin Johns, Hugh Tildesley
}

ß See related article page 505

In this first of 2 commentaries, James McCormack and coauthors present their view on whether the current contraindications to metformin therapy are warranted for certain patients with type 2 diabetes. We sent the article, which arrived unsolicited, out for peer review and received a particularly thoughtful assessment from George Fantus, who weighed some of the same evidence and reached a different conclusion (see page 505). We thought you might find it as interesting as we did to read 2 careful considerations of the same clinical question that reach different answers. Evidence, at least for this issue, is in the eye of the beholder. - CMAJ

M etformin has been used for over 40 years for patients with type 2 diabetes mellitus. ${ }^{1}$ With over 40 million patient-years of use as of $1999,{ }^{1}$ there is now evidence that the drug decreases the risk of morbidity and death when used to treat type 2 diabetes. ${ }^{2}$ However, concern remains over the possible side effect of lactic acidosis, a condition with a mortality of up to $50 \% .^{3}$ Because of this concern, contraindications to the use of metformin have been suggested. In particular, the following 3 are listed in the Compendium of Pharmaceuticals and Specialties: ${ }^{4}$

- Renal impairment, as suggested by elevated serum creatinine levels $(\geq 136 \mathrm{mmol} / \mathrm{L}$ in men and $\geq 124 \mathrm{mmol} / \mathrm{L}$ in women) or abnormal creatinine clearance ${ }^{4}$

- Congestive heart failure requiring pharmacologic treatment $^{4}$

- Advanced age ( $\geq 80$ years), unless measurement of creatinine clearance shows that renal function is not reduced ${ }^{5}$

These specific contraindications cause consternation for clinicians who may wish to prescribe metformin but do not want to put their patients at risk of lactic acidosis or expose themselves to potential legal problems. However, we believe the evidence shows that the benefits of metformin use in patients with contraindications outweigh the risks. In 2002 Calabrese and associates ${ }^{5}$ reported that $62 \%$ ऽิ of 204 hospital patients taking metformin had at least 1 contraindication or precautionary condition (renal impairment, congestive heart failure, age $\geq 80$ years, exposure to contrast media, hepatic disease, excessive alcohol intake, surgery). ${ }^{5}$ In particular, $14 \%$ of the patients taking metformin had an elevated creatinine level and 10\% were older than 80. These findings led Calabrese and associates

to state that "many patients are treated with metformin despite having clinical conditions that place them at risk for developing lactic acidosis. To minimize this risk, it is essential that prescribers develop a better understanding of the prescribing guidelines for metformin." Interestingly, despite the presence of these risks, lactic acidosis did not develop in any of the patients in their study. Although these authors' intentions were good, they and other clinicians who avoid metformin use in these patients, in our opinion, have not made an evidence-based decision. In a study from Germany that examined the prescribing of metformin outside of its clinical recommendations, 73\% of 308 patients had at least 1 contraindication to the drug; despite this, no cases of lactic acidosis were found..$^{14}$ In a study from Scotland involving 1847 patients taking metformin, the drug was prescribed outside of guidelines in $24.5 \%$ of cases; despite this, only 1 case of lactic acidosis occurred over a 30 -month period, and its cause was secondary to cardiac failure. ${ }^{15}$

An estimate of the absolute benefit of metformin use in terms of macrovascular end points can be taken from the results of the UK Prospective Diabetes Study (UKPDS). In this trial, absolute reductions were observed over 10 years in the number of diabetes-related deaths (by 5\%), all-cause mortality (by 7\%), myocardial infarction (by 6\%) and stroke (by $3 \%$ ) among patients with newly diagnosed type 2 diabetes who were given metformin compared with those following dietary advice. ${ }^{6}$ In the same study, the use of insulin and glyburide did not lead to any reductions in the risk of macrovascular disease compared with dietary advice alone. So far, the only other drug with any evidence of reducing macrovascular complications in type 2 diabetes is acarbose.

The conclusions from the UKPDS and from a recent meta-analysis have been debated..$^{8-10}$ It is important to remember, though, that the benefit from using metformin in the UKPDS was observed among patients with newly diagnosed type 2 diabetes, who would likely be at a lower absolute risk of diabetic complications than patients who have had the disease for a few years or have advanced renal or cardiovascular disease. ${ }^{11}$ Therefore, if anything, the absolute magnitude of the benefit of using metformin in "typical" patients with type 2 diabetes would likely be greater, because their initial absolute risk would be greater. 
The benefit of using metformin is fairly clear, but what is the risk of lactic acidosis among these patients? This is a more difficult question to answer. In a recent study by Salpeter and coauthors, ${ }^{12}$ pooled data from 194 studies showed no cases of lactic acidosis in over 35000 patientyears of metformin use. It must be remembered that this condition occurs in diabetic patients independent of metformin use. In 1998 Brown and colleagues ${ }^{13}$ reported on the incidence of lactic acidosis before and after the introduction of the drug in the United States: they found no difference in the incidence rates in 41000 patient-years. Before the drug's introduction, they placed the rate of lactic acidosis among patients not receiving metformin at 9.7-16.9 events per $100000 .{ }^{13}$ The meta-analysis by Salpeter and coauthors ${ }^{12}$ suggests that the upper limit for the true incidence of lactic acidosis is 9.9 events per $100000 \mathrm{pa}-$ tient-years among patients with type 2 diabetes not receiving metformin and 8.1 per 100000 among those taking the drug. In a historical cohort, Stang and colleagues $^{3}$ suggested a similar rate of lactic acidosis among metformin users at 9 cases per 100000 patient-years. This suggests that diabetes alone is an equally relevant, if not more relevant, risk factor for lactic acidosis than is metformin use.

In the trials included in the meta-analysis by Salpeter and coauthors, ${ }^{12} 16 \%$ of the patients were over the age of 65 years, and $44 \%$ of the trials allowed for the inclusion of patients with renal insufficiency, usually defined as a serum creatinine level of more than $132 \mathrm{mmol} / \mathrm{L}$. Of the studies, $96 \%$ allowed for the inclusion of patients with hypoxemic comorbidities such as renal insufficiency, cardiovascular disease, liver disease or pulmonary disease; however, these authors were unable to determine how many patients with these conditions were included in the clinical trials, and so it is not possible to determine the specific incidence of lactic acidosis among patients with these supposed contraindications. Nonetheless, the incidence of lactic acidosis in these studies was still zero.

Case studies have shown an association between reduced renal function, as indicated by elevated serum creatinine levels, and the incidence of lactic acidosis. ${ }^{11,16-18}$ Sulkin and coauthors ${ }^{17}$ described 2 patients with reduced renal function (serum creatinine levels 150 and $203 \mathrm{mmol} / \mathrm{L}$ ) in whom lactic acidosis developed while they were taking metformin. Misbin and colleagues ${ }^{19}$ found that, of $20 \mathrm{pa}-$ tients who died of lactic acidosis while taking metformin, $80 \%$ had significant renal impairment; the authors stated that lactate accumulation can result from renal impairment and its association with metformin use may have been coincidental. ${ }^{19}$ In addition, metformin-associated lactic acidosis can develop in patients with normal serum creatinine levels. There are published reports of 3 patients in whom lactic acidosis developed without there having been any indications of conditions that would increase the risk of this complication. ${ }^{20,21}$ All of the patients' hepatic, cardiac and renal functions were within normal limits, and all of the patients were taking normal doses of metformin (500 mg, $850 \mathrm{mg}$ and $1000 \mathrm{mg}$, respectively, twice daily). In 1 case, the woman was underweight and died; possible intentional overdose of metformin could not be ruled out.

In the other 2 cases, metformin therapy was stopped, and the patients recovered. ${ }^{20,21}$ An earlier case study described 6 patients with lactic acidosis whose serum creatinine levels ranged from 90 to $274 \mathrm{mmol} / \mathrm{L}$ before diagnosis and from 168 to $663 \mathrm{mmol} / \mathrm{L}$ after diagnosis; ${ }^{22}$ however, 3 of the patients, whose creatinine levels were normal, had undergone treatment involving contrast media. This may have been the reason for the development of lactic acidosis, although there is a lack of evidence to support a recommendation to withhold metformin therapy after the use of contrast media in patients with normal renal function. ${ }^{23}$

When the 79 cases of lactic acidosis in the literature are analyzed together, a unifying theme appears to emerge. Studies have shown that plasma metformin levels were not correlated to blood lactate levels, which raises the question of whether metformin is a causal factor in lactic acidosis. ${ }^{16,23}$ Lactic acidosis is associated with acute events such as myocardial infarction or congestive heart failure, acute renal insufficiency and sepsis. These 79 cases resulted after an estimated 1.1 million patient-years of metformin use, and in $37 \%$ of the cases the patient had a cardiac condition (myocardial infarction or congestive heart failure), in $24 \%$ an acute renal condition, in $8 \%$ an acute hepatic condition and in $4 \%$ sepsis. In all of these cases the underlying conditions could have themselves caused the lactic acidosis, so it is difficult to identify the degree to which metformin was responsible.

There is little, if any, research exploring rates of lactic acidosis among metformin users with rates among users of other oral hypoglycemic agents. ${ }^{3}$ It appears that the incidence of metformin-associated lactic acidosis is not much different than the baseline incidence among people with type 2 diabetes. Even if the presence of renal failure in- 
creased the incidence of lactic acidosis 10-fold over baseline, the incidence would still be only about $1 \%$ over 10 years (with a baseline rate of 10 per 100000 patient-years, the 10 -fold increase would result in a rate of 100 per 100000 patient-years, which would be about 1 per 1000 patient-years, or $1 \%$ over 10 years). The benefit of using metformin would be a reduction of $5 \%$ in diabetes-related deaths, $7 \%$ in all-cause mortality, $6 \%$ in myocardial infarction and $3 \%$ in stroke.

Given the above information, it is clear that, even among patients with the so-called "contraindications," the magnitude of the benefit of metformin therapy would clearly outweigh any potential risk. What, then, should clinicians do about this issue? They need to decide whether the above evidence warrants a discussion about the specific risk of lactic acidosis. If the issue of lactic acidosis is of enough concern to them, this issue should be discussed not only with patients taking metformin, but also with any patient who has type 2 diabetes, because the risk is really not a lot different. Perhaps a reasonable approach is to tell patients, as one should when prescribing any drug therapy, that if any unexplained symptoms develop such as nausea or vomiting, abdominal pain, rapid breathing, difficulty breathing, chest pain, weakening of the muscles in the legs and arms, diarrhea, skin rash and confusion, one of the potential reasons for these symptoms could be the drug but it could also possibly mean another medical condition. Regardless, if these symptoms occur, patients should seek prompt medical attention.

Using metformin in a patient of advanced age $(\geq 80$ years) or in a patient who has reduced renal function requires one to consider the potential for decreased elimination ability. Unfortunately for metformin, there really is no solid evidence to guide clinicians as to what to do. A reasonable approach might be, as with all patients, to start with a low dose ( $250 \mathrm{mg}$ twice daily) and increase the dose weekly, based on tolerance and effect of the drug on the surrogate end point of blood glucose level, to a maximum dose of $2000-2500 \mathrm{mg} / \mathrm{d}$. Based on general pharmacokinetic principles, rather than avoid the drug completely, it would be reasonable to reduce the maximum dose by about $50 \%$ in a patient with an estimated creatinine clearance of less than $1.0 \mathrm{~mL} / \mathrm{s}$.

In conclusion, the evidence at present suggests that the use of metformin in patients who are over the age of 80 years, have congestive heart failure or have renal insufficiency leads to a benefit that far outweighs the potential harm. We would suggest that it is a "contraindication" to not use metformin in people with type 2 diabetes with these contraindications.

This article has been peer reviewed.

From the Faculty of Pharmaceutical Sciences, University of British Columbia (McCormack); and the Division of Endocrinology, St. Paul's Hospital, Vancouver, BC (Johns, Tildesley).

Competing interests: None declared.
Contributors: Hugh Tildesley, the chief investigator of the project, drafted portions of the article, provided relevant background information, contributed intellectual content and gave final approval. Kevin Johns performed the literature search, was the primary author of the article and gave final approval. James McCormack, the co-investigator of the project, interpreted data, drafted portions of the article, critically revised the article for important intellectual content and gave final approval.

\section{References}

1. Howlett HCS, Bailey CJ. A risk-benefit assessment of metformin in type 2 diabetes mellitus. Drug Saf 1999;20:489-503.

2. UK Prospective Diabetes Study (UKPDS) Group. Effect of intensive bloodglucose control with metformin on complications in overweight patients with type 2 diabetes (UKPDS 34). Lancet 1998;352:854-65.

3. Stang M, Wysowski DK, Butler-Jones D. Incidence of lactic acidosis in metformin users. Diabetes Care 1999;22:925-7.

4. Canadian Pharmacists Association. Compendium of pharmaceuticals and specialties. Ottawa: The Association; 2001.

5. Calabrese AT, Coley KC, DaPos SV, Swanson D, Rao RH. Evaluation of prescribing practices: risk of lactic acidosis with metformin therapy. Arch Intern Med 2002;162:434-7.

6. McCormack J, Greenhalgh T. Seeing what you want to see in randomised controlled trials: versions and perversions of UKPDS data. BMF 2000;320: 1720-3.

7. Chiasson JL, Josse RG, Gomis R, Hanefeld M, Karasik A, Laakso M; STOPNIDDM Trial Research Group. Acarbose treatment and the risk of cardiovascular disease and hypertension in patients with impaired glucose tolerance: the STOP-NIDDM trial. FAMA 2003;290:486-94.

8. Van de Laar FA, Lucassen PL, Akkermans RP, van de Lisdonk EH, Rutten GE, van Weel C. [alpha]-Glucosidase inhibitors for patients with type 2 diabetes: results from a Cochrane systematic review and meta-analysis. Diabetes Care 2005;28:154.

9. Hanefeld M, Cagatay M, Petrowitsch T, Neuser D, Petzinna D, Rupp M Acarbose reduces the risk for myocardial infarction in type 2 diabetic patients: meta-analysis of seven long-term studies. Eur Heart 7 2004;25:10-6.

10. Van de Laar FA, Lucassen PL. No evidence for a reduction of myocardial infarctions by acarbose [letter]. Eur Heart 7 2004;25:1179.

11. Intensive blood-glucose control with sulphonylureas or insulin compared with conventional treatment of complications in patients with type 2 diabetes (UKPDS 33). Lancet 1998;352:837-53.

12. Salpeter SR, Greyber E, Pasternak GA, Salpeter EE. Risk of fatal and nonfatal lactic acidosis with metformin use in type 2 diabetes mellitus. Arch Intern Med 2003;163:2594-602.

13. Brown JB, Pedula K, Barzilay J, Herson MK, Latare P. Lactic acidosis rates in type 2 diabetes. Diabetes Care 1998;21:1659-63.

14. Holstein A, Nahrwold D, Hinze S, Egberts EH. Contra-indications to metformin therapy are largely disregarded. Diabet Med 1999;16:692-6.

15. Emslie-Smith AM, Boyle DIR, Evans JMM, Sullivan F, Morris AD. Contraindications to metformin therapy in patients with type 2 diabetes - a population-based study of adherence to prescribing guidelines. Diabet Med 2001; 18:483-8.

16. Lalau JD, Lacroix C, Compagnon P, de Cagny B, Rigaud JP, Bleichner G, et al. Role of Metformin Accumulation in metformin-associated lactic acidosis. Diabetes Care 1995;18:779-84.

17. Sulkin TV, Bosman D, Krentz AJ. Contraindications to metformin therapy in patients with NIDDM. Diabetes Care 1997;20:925-8.

18. Waters AK, Morgan DB, Wales JK. Blood Lactate and pyruvate levels in diabetic patients treated with biguandes with and without sulphonylureas. Diabetologia 1978;14:95-8.

19. Misbin RI, Green L, Stadel BV, Gueriguian JL, Gabbi A, Fleming GA. Lactic acidosis in patients with diabetes treated with metformin. N Engl $7 \mathrm{Med}$ 1998;338:265-6.

20. Ellis AK, Iliescu EA. Metformin-associated lactic acidosis in a low risk patient. Can 7 Clin Pharmacol 2001;8:104-6.

21. Khan JK, Muralidhar P, Tolber SR, Hornick TR. Lactic acidemia associated with metformin. Ann Pharmacother 2003;37:66-9.

22. Assan R, Heuclin C, Ganeval D, Bismuth C, George J, Richard JR. Metformin-induced lactic acidosis in the presence of acute renal failure. Diabetologia $1977 ; 13: 211-7$

23. Jones GC, Macklin JP, Alexander WD. Contraindications to the use of metformin. BM7 2003;326:4-5.

Correspondence to: Dr. James McCormack, Endocrine Research, St. Paul's Hospital, 467-1081 Burrard St., Vancouver BC V6Z 1Y6; fax 604 631-5154; jmccorma@interchange.ubc.ca 